\title{
Germes et cavitation Effets - Techniques de contrôle - Résultats récents
}

\author{
Y. Lecoffre Bassin des carènes - Le Val de Reuil \\ L. Menoret Alsthom ACB, CERG - Pont de Claix \\ L. Briançon-Marjollet, J.P. Franc, J.M. Michel Institut de mécanique de Grenoble
}

Parmi les nombreux facteurs qui influent sur la cavitation, la qualité de l'eau joue un rôle fondamental en raison des germes qu'elle peut contenir. Il est donc important de contrôler et de caractériser la teneur de l'eau en germes de cavitation dans les moyens d'essais.

On décrit tout d'abord la technique d'injection et de mesure de germes développée par le CERG. Les principales caractéristiques du système sont présentées à partir de mesures réalisées sur les tunnels à cavitation du CERG et de l'IMG. Les limitations actuelles du dispositif ainsi que les principaux problèmes rencontrés dans son utilisation sont discutés.

Enfin, on montre à partir d'observations réalisées sur profils (ailes et ogives), l'effet de l'injection de germes sur la cavitation naissante et le type de cavitation qui se développe (par poche attachée ou par bulles séparées).

\section{Introduction}

Dans la théorie classique des écoulements cavitants, le paramètre de cavitation $\sigma_{v}$ joue un rôle prépondérant. Il est défini par:

$$
\sigma_{v}=\frac{p_{\infty}-p_{v}}{1 / 2 \rho V_{\infty}^{2}}
$$

où $p_{\infty}$ et $V_{\infty}$ désignent une pression et une vitesse de référence et $p_{v}$ la pression de vapeur du liquide.

\begin{abstract}
On admet classiquement que, si la similitude en $\sigma_{v}$ est assurée entre deux écoulements par ailleurs géométriquement semblables (modèle et prototype par exemple), l'extension de la zone cavitante ainsi que les divers paramètres hydrodynamiques tels que les coefficients d'efforts sont également en similitude.

Pourtant, des déviations importantes par rapport à cette règle élémentaire ont été depuis longtemps observées (Holl \& Wislicenus 1961 - Arndt 1981). En ce qui concerne la naissance de la cavitation par exemple, des écarts ont été mis en évidence entre la valeur $\sigma_{v i}$ du paramètre de cavitation à partir de laquelle les premières
\end{abstract}

\section{Nuclei \& Cavitation : effects - control techniques - recent results.}

Among the numerous factors which influence cavitation, the quality of water plays a fundamental role because of the nuclei it may contain. Thus it is important to control and to characterize the cavitation nucleus content of water in experimental facilities.

Firstly we describe the techniques of injection and measurement of nuclei developed by CERG. The main characteristics of the system are presented from measurements which were carried out on the cavitation tunnels of CERG and IMG. Present limitations of the system and the main difficulties raised by its use are discussed.

Finally we show, on the basis of observations carried out on foils and ogives, the effect of nucleus injection on cavitation inception and cavitation patterns which develop (attached civity or travelling bubbles). 


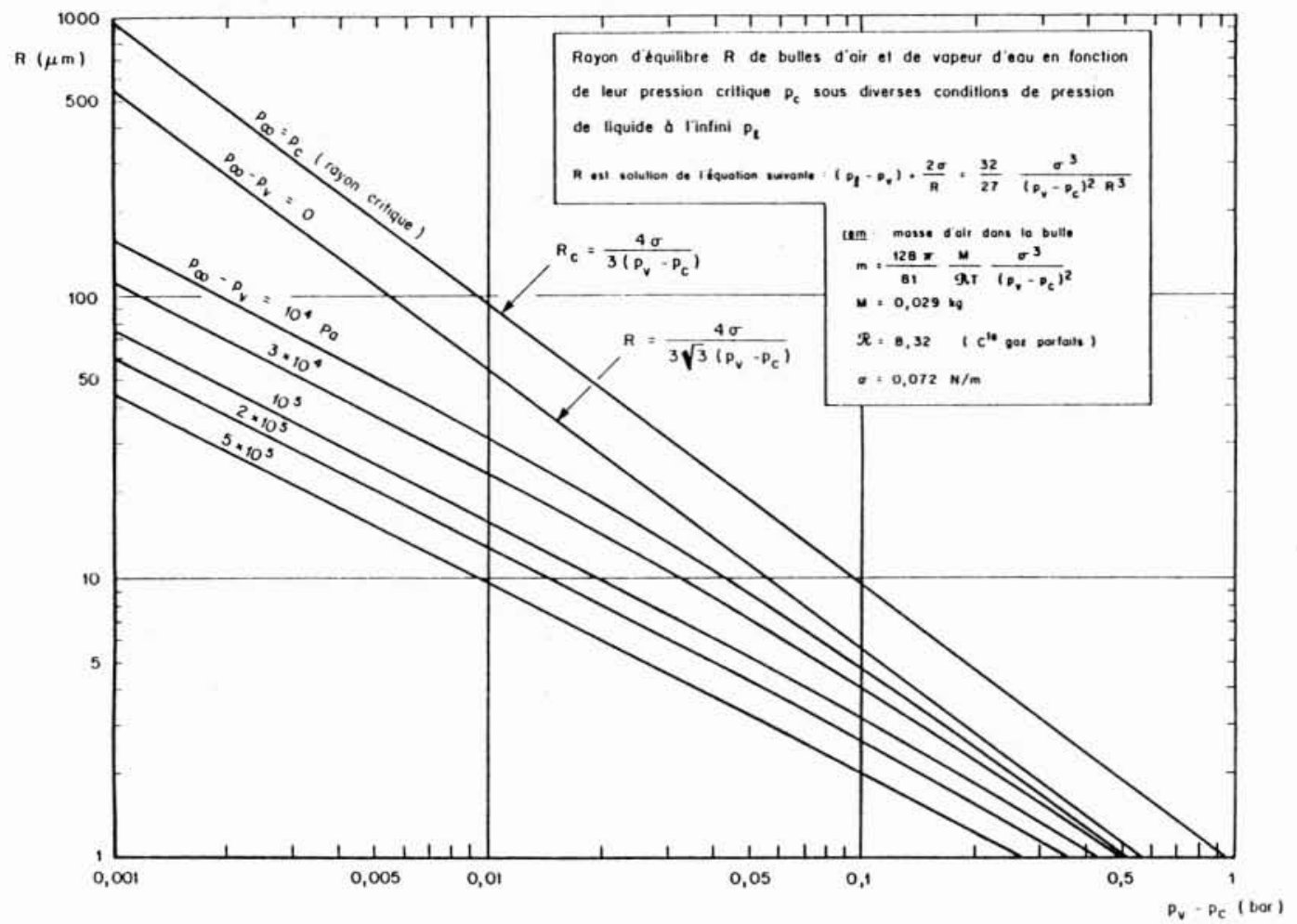

figures de cavitation apparaissent et sa valeur théorique, égale à l'opposé du coefficient de pression minimal $-C_{p \text { mini; }}$ une influence sensible du nombre de Reynolds et de l'échelle géométrique sur le paramètre de cavitation naissante a également été observée par de nombreux expérimentateurs. Le type même de cavitation peut dépendre du moyen d'essais utilisé, voire des conditions opératoires (Lindgren \& Johnson 1966).

Ces déviations sont attribuées aux paramètres physiques que ne prend pas en compte le schéma du fluide non visqueux en écoulement irrotationnel sur lequel repose la règle de similitude en $\sigma_{v}$. Parmi ces paramètres, on peut citer les propriétés physiques du fluide et de sa vapeur (viscosité, tension superficielle, propriétés thermiques), la rugosité des parois solides, le taux de turbulence général de l'écoulement et la qualité du liquide caractérisée par sa teneur en germes de cavitation qui fait l'objet de la présente étude.

Dans le cas de l'eau froide auquel nous nous limitons ici, les germes sont principalement constitués par des microbulles de gaz. Celles-ci sont entraînées par l'écoulement, et certaines traversent les zones de basse pression, critiques pour la cavitation. Selon leurs caractéristiques, elles peuvent traverser ces zones sans subir d'évolution sensible ou au contraire y exploser et donner naissance à des bulles de cavitation macroscopiques.
Des essais réalisés sur divers types de machines ou d'organes hydrauliques ont mis en évidence l'influence de la qualité de l'eau sur la cavitation. Henry \& al. (1980) et Avellan \& al. (1986) ont montré que l'introduction de germes dans l'écoulement modifie le début de cavitation et les performances en cavitation plus développée des modèles de turbines Francis. D'autres résultats comparables ont été obtenus sur des hélices supercavitantes (Suhrbier \& Lecoffre - 1986) et sur des vannes (Lecoffre - 1986).

Il est donc important de pouvoir contrôler la teneur de l'eau en germes de cavitation dans les moyens d'essais. Cela nécessite de disposer d'une part d'un système de mesure permettant de qualifier le contenu de l'eau en germes, et d'autre part d'une technique d'injection de germes permettant de modifier la qualité de l'eau.

Après un bref rappel de quelques généralités sur les germes de cavitation, nous présentons les techniques de contrôle de germes utilisées en moyens d'essais, et en particulier les dispositifs d'injection et de mesure développé par le CERG. Nous donnons ensuite quelques résultats qui ont été obtenus récemment sur les tunnels hydrodynamiques du CERG et de l'IMG, tous deux équipés d'un tel dispositif, et qui illustrent en particulier l'influence de la teneur de l'eau en germes sur le type de cavitation, par poche attachée ou par bulles séparées. 
2. Généralités sur les germes de cavitation : Stabilité - Histogramme - Similitude

\subsection{Pression critique et histogramme des pressions critiques}

Pour qu'un germe grossisse de façon explosive et passe ainsi d'une taille micrométrique à une taille millimétrique, il faut lui appliquer une pression inférieure à une pression critique $p_{c}$ variable d'un germe à un autre, elle-même inférieure à la pression de vapeur saturante $p_{v}$.

Le rayon d'équilibre $R$, sous la pression $p_{\ell}$ de liquide, d'une microbulle contenant de la vapeur d'eau à la pression de vapeur saturante $p_{v}$ et du gaz incondensable dont la pression partielle dans la bulle est $p_{g}$, est donné par la relation suivante :

$$
p_{\ell}=p_{v}+p_{g}-(2 S / R)
$$

où $S$ désigne la tension superficielle.

Si le germe grossit par vaporisation, la masse de gaz occlus reste constante. $\mathrm{Si}$, de plus, le gaz subit une transformation isotherme et en l'assimilant, en première approximation à un gaz parfait, sa pression partielle $p_{g}$ est inversement proportionnelle au volume de la bulle.

Dans ces conditions, lorsque la pression $p_{\ell}$ du liquide diminue suffisamment lentement, le germe initialement en équilibre grossit et son rayon atteint pour chaque pression une valeur d'équilibre qui satisfait l'équation (1). Si la pression tombe en-dessous d'une valeur critique $p_{c}$, il n'existe plus de rayon d'équilibre satisfaisant l'équation (1): le germe grossit indéfiniment sans jamais retrouver de taille d'équilibre.

La figure 1 donne la pression critique d'un germe en fonction de son rayon d'équilibre sous différentes pressions $p_{1}$. En particulier, si le germe a un rayon $R$ lorsqu'il est en équilibre à la pression $p_{1}=p_{v}$, sa pression critique est donnée par la formule suivante:

$$
p_{c}=p_{v}-\frac{4}{3 \sqrt{3}} \frac{S}{R}
$$

Cette formule, du fait de sa simplicité, est généralement utilisée pour évaluer la taille d'un germe dont on connaît la pression critique.

A titre d'exemple, pour faire exploser un germe ayant un rayon de $20 \mu \mathrm{m}$ à la pression de vapeur saturante, il faut lui appliquer une tension correspondant à une pression négative de $-30 \mathrm{mb}$. Plus un germe est gros, plus sa pression critique est proche de $p_{v}$.

Ainsi, chaque germe peut être caractérisé par sa pression critique, et un volume donné d'eau est caractérisé par l'histogramme des pressions critiques des germes qu'il contient (figure 2). Nous verrons au paragraphe 3 comment on peut, en pratique, déterminer l'histogramme des pressions critiques d'une eau.

Dans un écoulement, seuls les germes dont la pression critique est supérieure à la pression minimale de l'écoulement pourront être activés et exploser.

L'approche qui vient d'être présentée repose sur un modèle d'évolution quasi-statique. Dans certains cas, par

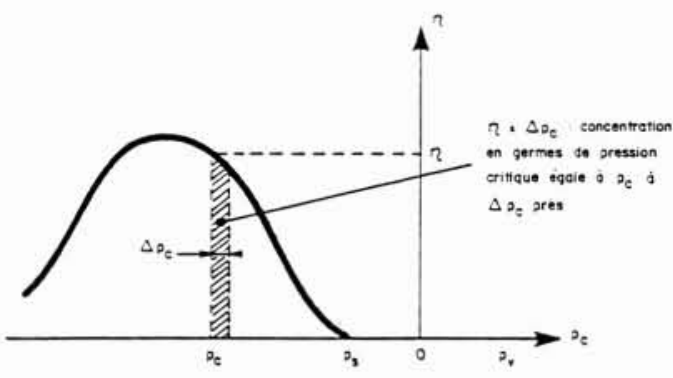

2. Histogramme des pressions critiques.

exemple pour des profils à grande incidence présentant un pic de dépression profond et localisé, il se peut que les effets dynamiques prennent de l'importance. On préfère dans ce cas le terme de pression d'éclatement au terme de critique, ce dernier étant plutôt réservé à l'approche quasi-statique. Il convient alors d'utiliser l'équation de Rayleigh-Plesset (voir par exemple Plesset \& Prosperetti - 1977) qui gouverne l'évolution temporelle du rayon de la bulle. Néanmoins, le modèle quasi-statique constitue, du fait de sa simplicité, le modèle de base pour l'étude des germes de cavitation.

\subsection{Similitude en cavitation à bulles séparées}

\section{Cavitation naissante}

L'aptitude du liquide à caviter dépend de la pression critique la plus grande $p_{s}$ des germes qu'il contient. Un écoulement commence à caviter sous la forme de bulles séparées dès que la pression minimale atteint la pression critique $p_{s}$ caractérisant la susceptibilité du liquide, du moins si les retards dus à l'inertie sont négligeables.

Le paramètre de cavitation naissante $\sigma_{i}$ est alors donné par la formule suivante

$$
\sigma_{i}=-C_{p \operatorname{mini}}-\Delta \sigma, \Delta \sigma=\frac{p_{v}-p_{s}}{1 / 2 \rho V_{\infty}^{2}}
$$

Le retard $\Delta \sigma$ à la cavitation est d'autant plus faible que l'eau contient des gros germes et que la vitesse de l'écoulement est grande comme le montre la figure 3 .

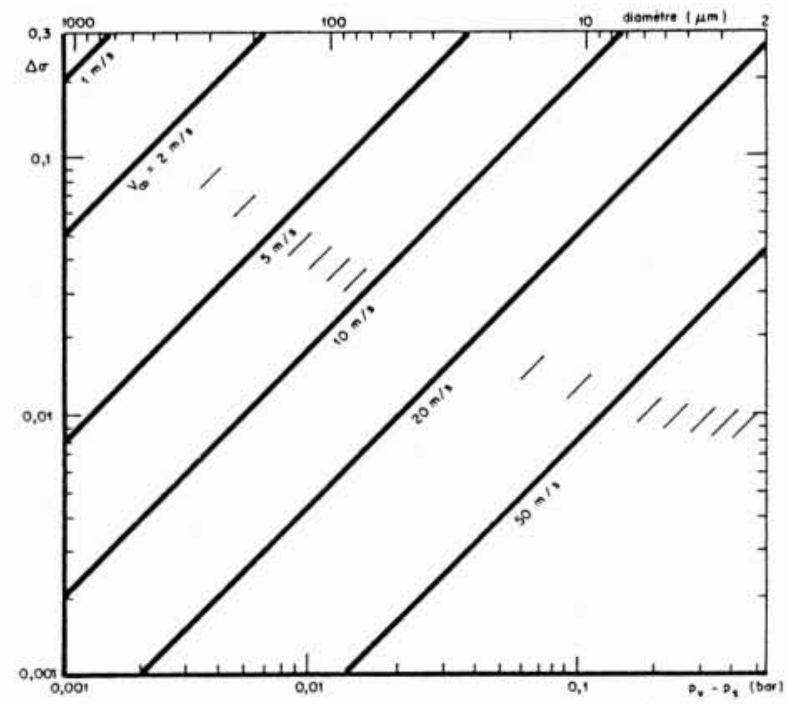

3. Retard à l'apparition de la cavitation $\Delta \sigma$ en fonction de la vitesse $V_{\infty}$ et du diamètre maximal des germes à la pression de vapeur saturante ou de leur pression critique maximale. 


\section{Cavitation développée}

Pour assurer une parfaite similitude en cavitation à bulles séparées, il faudrait théoriquement assurer la similitude complète des histogrammes de pressions critiques. En pratique cette condition est difficile, voire impossible, à réaliser et de toute façon il n'est pas nécessaire de réaliser une similitude aussi fine.

En effet, seuls les germes actifs ont une importance, les autres ne grossissant pas. Par ailleurs, si un germe explose, dès que son rayon atteint une taille de l'ordre du dixième de millimètre, la tension superficielle, la viscosité et les incondensables occlus ont un effet négligeable sur sa croissance. Dans le cas où la pression $p$ à laquelle il est soumis est constante, ce qui est pratiquement le cas des profils minces à faible incidence, son rayon augmente à la vitesse limite :

$$
\dot{R}=\sqrt{\frac{2}{3} \frac{p_{v}-p}{p}}=\sqrt{\frac{-C_{p}-\sigma_{v}}{3}} V_{\infty}
$$

On peut montrer en adimensionnalisant l'équation de Rayleigh-Plesset ainsi simplifiée que, pourvu que le germe explose et que la similitude en $\sigma_{v}$ soit réalisée, sa taille finale est indépendante de sa taille initiale (qui est négligeable) et de la vitesse de l'écoulement, et est proportionnelle à l'échelle géométrique. Dans le cas simplifié où il traverse une zone de dépression constante de longueur $L$ à la vitesse locale $V=V_{\infty} \sqrt{1-C_{p}}$, sa taille finale est, d'après (4), donnée par :

$$
\frac{R_{\max }}{L}=\sqrt{\frac{-C_{p}-\sigma_{v}}{3\left(1-C_{p}\right)}}
$$

Cette formule montre clairement que la taille des bulles de cavitation ne dépend, en première approximation, que de l'échelle géométrique, pour des écoulements géométriques semblables à paramètre de cavitation fixé.

Pour assurer la similitude des figures de cavitation développée par bulles, il suffit donc d'assurer, outre l'égalité des paramètres de cavitation qui permet une croissance semblable de germes, l'égalité des nombres totaux de germes actifs. Si les échelles géométriques sont dans le rapport $\lambda$, les concentrations volumiques en

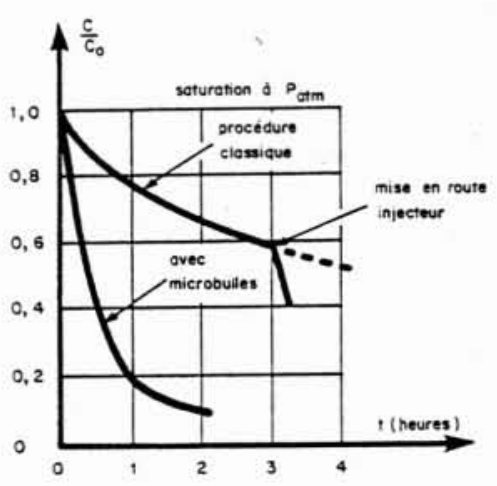

4. Dégazage accéléré par injection de microbulles (tunnel TH8). germes actifs doivent être dans le rapport $1 / \lambda^{3}$ comme l'ont proposé Holl \& Wislicenus (1961) et Le Goff \& Lecoffre (1982). Cette règle de similitude " en $\lambda^{3}$ " impose que la concentration en germes pour un essai sur modèle réduit soit généralement nettement supérieure à celle correspondant à l'écoulement prototype.

Lorsque leur nombre augmente beaucoup, les bulles interagissent et leur croissance s'en trouve limitée. On aboutit alors à un phénomène de saturation qui a été constaté par Avelan \& al. (1986) à partir de mesures de rendement sur des modèles de turbines Francis. Au-delà du seuil de saturation qui doit obéir à la loi en $\lambda^{3}$, les performances en cavitation ne sont plus affectées par la concentration en germes.

\section{Techniques de contrôle de la concentra- tion de l'eau en germes de cavitation}

\subsection{Principe}

Le principe du contrôle de la nucléation développé par le CERG est le suivant :

- On dégaze la boucle d'essais jusqu'à un niveau de concentration tel qu'aucune bulle ne fasse plus d'un tour de circuit.

- On injecte alors des microbulles en quantité et dimensions réglables à l'amont de la veine d'essais.

- On mesure, au moyen de Venturi spéciaux, le nombre et les dimensions des germes créés.

\subsection{Réglage de la teneur en air dissous}

La méthode traditionnelle de réglage de la teneur en air dissous consiste à mettre le circuit d'essais sous vide en faisant éventuellement caviter le composant testé.

On peut notablement accélérer ce processus en créant, dans les zones de basse pression de l'installation, des surfaces d'échange importantes.

Si l'on appelle $m$ la masse totale de gaz dissous présente dans une installation à l'instant $t, P_{\text {sat }}$ la pression de saturation du liquide et $P$ la pression régnant dans la zone de dégazage, le débit libéré s'écrit :

$$
\frac{d m}{d t}=K_{L} \cdot S \cdot H \cdot\left(P_{\text {sat }}-P\right)
$$

où $H$ est la constante de Henry et $K_{L}$ le coefficient d'échange.

Il est donc directement proportionnel à $S$, surface d'échange gaz-liquide disponible.

Une technique récemment développée au CERG consiste à injecter dans la zone de pression une émulsion de microbulles obtenue par détente d'eau préalablement sursaturée. L'émulsion est formée de bulles de gaz de quelques dizaines de microns de diamètre qui, lors de leur transport par l'écoulement sont autant de sites de dégazage.

On donne, à titre d'exemple (fig. 4), l'évolution de la teneur en gaz dissous dans un tunnel de cavitation du CERG avec et sans mise en œuvre du système. On 
constate que le temps nécessaire pour passer de la saturation Co sous pression atmosphérique à $20 \%$ de celle-ci est réduit à 1 heure environ contre $40 \mathrm{~h}$ sous une procédure classique.

Un système analogue est utilisé sur la boucle EPOCA de l'E.D.F. Un autre est mis en œuvre sur le tunnel GTH du Val de Reuil dont le volume d'eau est $3600 \mathrm{~m}^{3}$.

Cette méthode, qui présente l'avantage d'utiliser la boucle d'essai elle-même comme dégazeur, n'est bien sûr intéressante que quand le dégazage naturel requiert des temps d'immobilisation de plateformes inacceptables.

\subsection{Réglage de la teneur en gaz libre}

La concentration en germes efficaces sur modèle doit pouvoir être égale, selon l'échelle du modèle, à des valeurs comprises classiquement entre 1 et 100 par $\mathrm{cm}^{3}$, les bulles produites ayant quelques dizaines de microns de diamètre.

La technique utilisée au CERG consiste à faire se détendre de l'eau initialement saturée sous pression au travers d'un orifice de très petites dimensions. La cavitation produite dans cet orifice donne naissance à un grand nombre de microbulles. On obtient, par exemple, un débit de bulles de $5.10^{6} / \mathrm{s}$ pour une pression dans le réservoir d'engazage de 20 bars et une pression dans le tunnel de 3 bars. Les bulles produites sont d'autant moins nombreuses et d'autant plus grosses que la pression dans le tunnel est basse.

La figure 5 montre une rampe de générateurs en fonctionnement dans un canal à surface libre. Le débit de bulles produites est, dans ce cas, de $3.10^{7}$ par seconde environ. Le débit d'eau saturée est de 1,5 l/s.

Dans certaines applications, il est nécessaire de réduire les concentrations en bulles tout en continuant à les contrôler. On utilise alors des générateurs à faible débit et l'on dilue les bulles qu'ils produisent dans de l'eau dépourvue de germes. C'est le cas des installations mises en œuvre sur le TH2 du CERG ou le tunnel de l'IMG (figure 7).

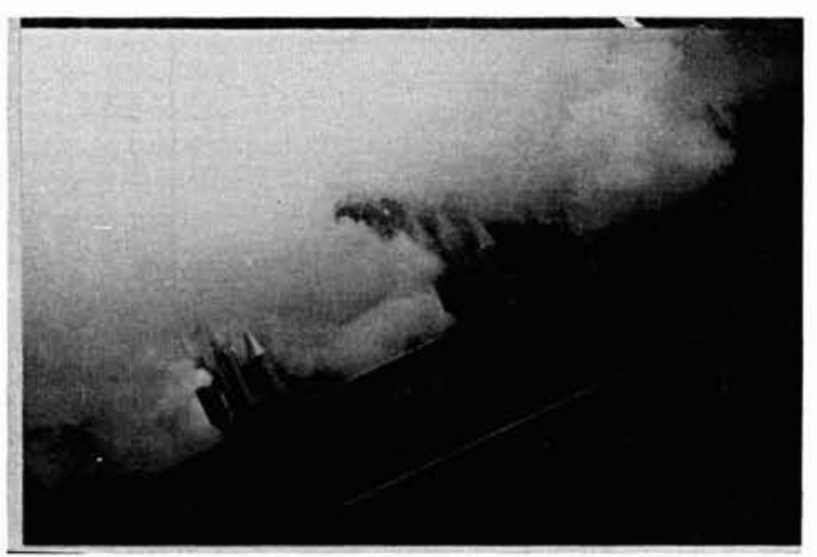

5. Rampe d'injecteurs de germes.

\subsection{Mesure des germes}

On utilise pour mesurer les germes de cavitation des méthodes cavitantes (microventuri) et des méthodes indirectes, optiques, acoustiques et électriques. L'objectif est de connaître la distribution (nombre, pression critique) du spectre de germes. Parmi les méthodes indirectes, la plus utilisée actuellement est la diffusion de la lumière (Billet 1985). On citera également l'holographie que l'on peut utiliser comme méthode de référence.

Les venturi de mesures de germes ont été développés à l'origine par Oldenziel (1976), puis par le CERG. Ces premiers appareils présentaient quelques défauts. Leur réalisation était très délicate. Les venturi CERG étaient réalisés par électro-déposition de nickel sur une forme interne usinée et polie. Par aileurs, le développement et la disparition des bulles de vapeur au col engendrait des pulsations de pression indésirables dans les tuyauteries d'alimentation qui pouvaient perturber la mesure lorsque les concentrations en germes dépassaient certaines valeurs seuil, de l'ordre de 1 germe par $\mathrm{cm}^{3}$. Enfin, pour certaines applications, le débit analysé de l'ordre de $0,1 \mathrm{l} / \mathrm{s}$ était insuffisant.

Les venturi récemment développés par Y. Lecoffre au CERG gardent le même principe de base, à savoir la création d'une zone de pression négative par mise en vitesse de l'écoulement. Ils sont formés d'un cône à grand angle $\left(20^{\circ}\right)$ à l'intérieur duquel se trouve une ogive. Dans la configuration actuellement utilisée, le jeu ogive-cône est de l'ordre de $1 \mathrm{~mm}$. Le débit nominal dans le venturi est de l'ordre de $1 \mathrm{l} / \mathrm{s}$, soit 10 fois supérieur à celui des venturi précédents. Le diamètre au col, à l'amont de l'ogive, est de $10,4 \mathrm{~mm}$.

Cette configuration permet d'éliminer la plupart des problèmes qu'avaient les précédents appareils :

- La tuyauterie amont est de grande dimension et le diamètre maximal des bulles est très limité. De ce fait, les pulsations de pression sont négligeables.

- Le passage étant quasi bidimensionnel, il n'y a que très peu d'interactions entre bulles, même lorsque plusieurs d'entre elles grossissent simultanément dans le venturi.

- La fabrication et l'entretien des appareils est beaucoup plus simple que précédemment. On peut les réaliser en plexiglas, et de ce fait visualiser les phénomènes.

- On constate enfin pratiquement que les réglages fins et en particulier la détermination précise des débuts de cavitation se font aisément.

L'appareil déjà longuement utilisé au CERG et à l'IMG, est en cours de qualification complète, celle-ci incluant des mesures précises de distribution de pression moyenne et de fluctuation de pression turbulente au col. La figure 6 montre l'allure des bulles produites au col pour deux conditions de fonctionnement différentes.

La mesure des germes est réalisée en continu grâce à des cannes de prélèvement. Celles-ci assurent un prélèvement de l'eau du tunnel qui est détournée vers le venturi de mesure. Un système de dilution avec de l'eau du tunnel exempte de germes permet la mesure de fortes concentrations de germes. La figure 7 donne un schéma de principe de l'ensemble des circuits d'injection et de mesure qui équipent le tunnel de l'IMG. 

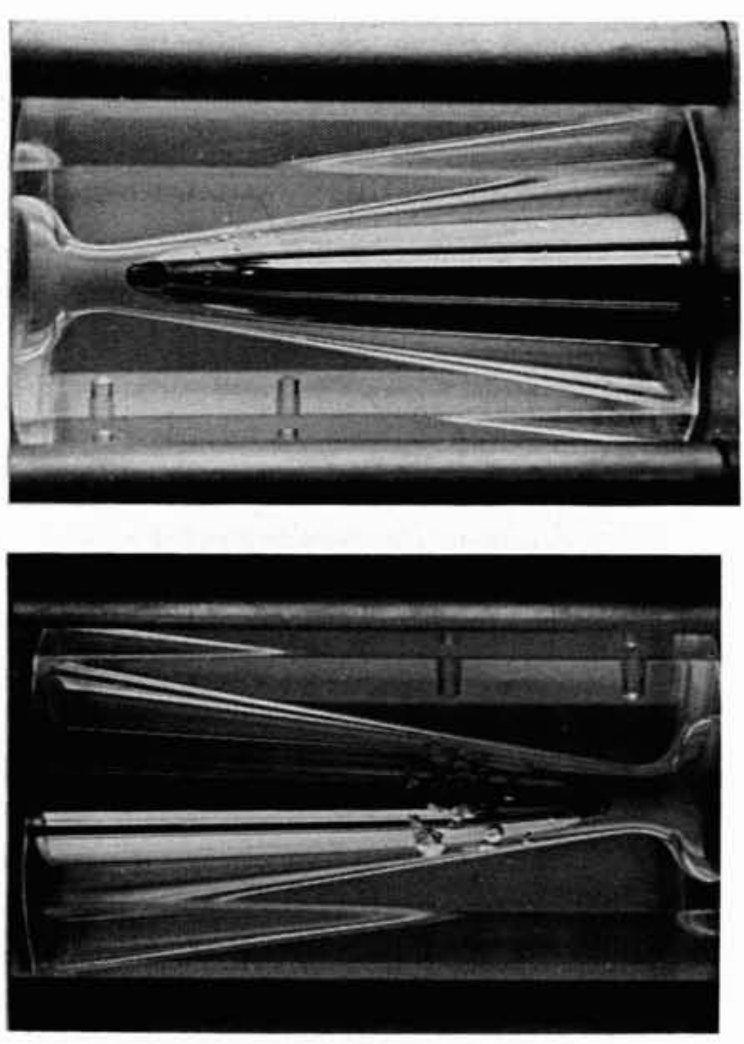

6. Venturi à ogive centrale.

8. Histogrammes cumulés de pressions d'éclatement, tous injecteurs ouverts (tunnel de l'IMG).

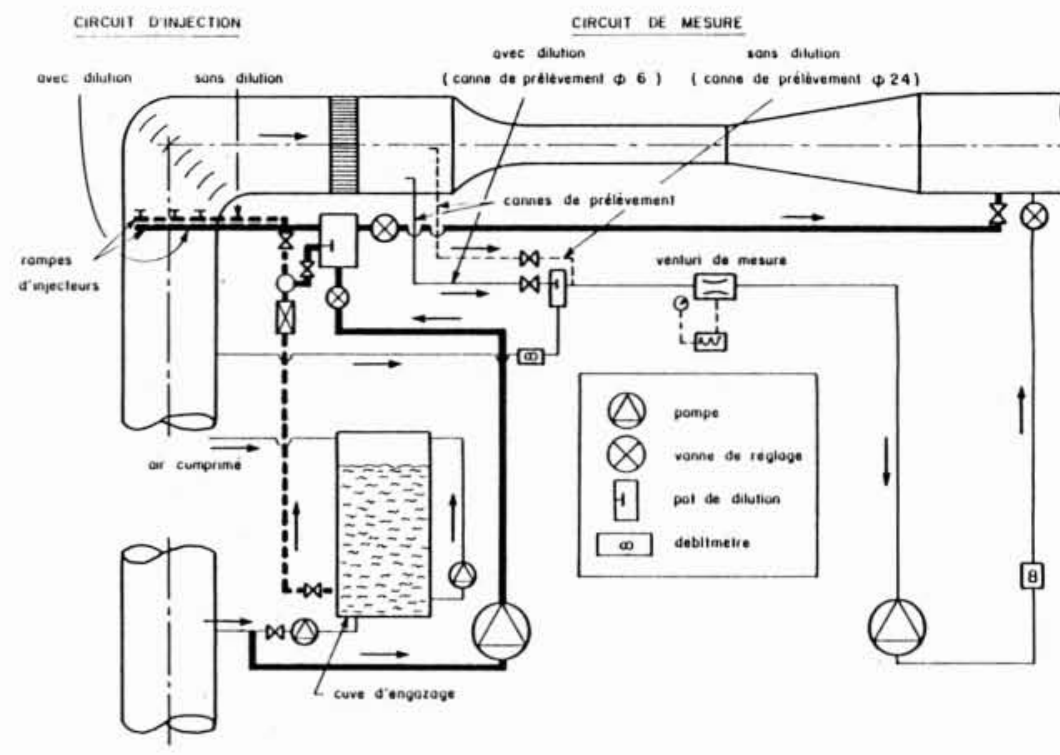

7. Schéma du dispositif de contrôle de la teneur de l'eau en germes de cavitation (tunnel de l'TMG).

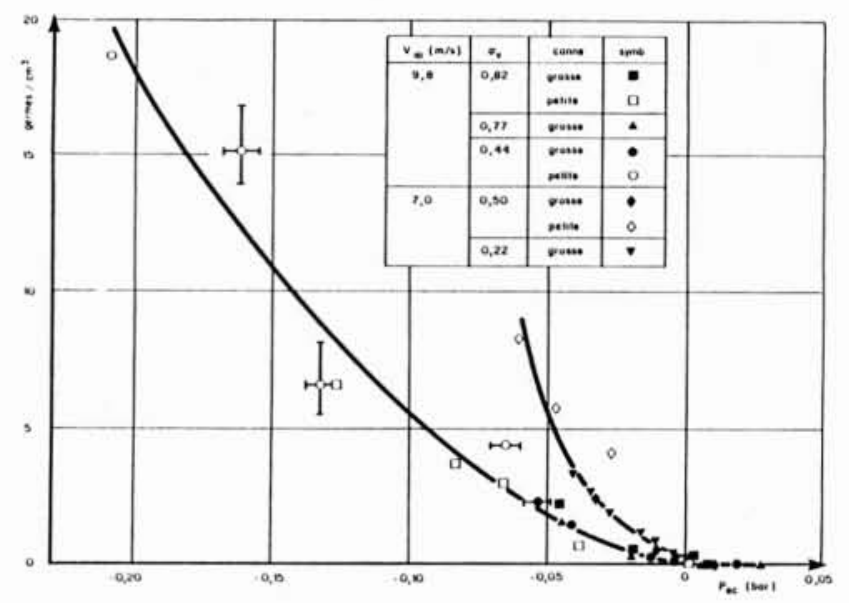

\subsection{Principaux problèmes posés par l'utilisa- tion du dispositif et ses limitations}

La figure 8 présente un exemple d'histogrammes cumulés donnant la concentration volumique des germes dont la pression d'éclatement est supérieure à une valeur donnée. Ils ont été obtenus sur le tunnel de l'IMG, tous injecteurs ouverts, sous diverses conditions de fonctionnement du tunnel. Il apparaît que, à vitesse donnée, la population de germes est pratiquement indépendante du paramètre de cavitation du tunnel, c'est-à-dire de sa pression de fonctionnement. Par contre, la concentration en germes a tendance à augmenter lorsque la vitesse diminue, principalement du fait d'une moindre dilution par l'eau du tunnel.
La densité maximale mesurable est de l'ordre de 20 germes $/ \mathrm{cm}^{3}$. La limitation essentielle est due à la fréquence de comptage qui ne peut pas dépasser 1500 à 2000 germes/s environ, valeur au-delà de laquelle le comptage se trouve affecté par un phénomène de saturation. Ce seuil conditionne également le passage de la grosse canne de prélèvement à la petite et l'utilisation de la dilution dans le circuit de mesure.

En ce qui concerne la pression d'éclatement, la limitation principale est due à l'apparition dans le venturi de mesure d'une cavitation par poche attachée qui rend impossible le comptage des germes. Des essais menés à l'IMG sous des conditions variées de fonctionnement du tunnel ont montré que la poche apparaît pour une valeur limite $\sigma_{1}$ pratiquement constante de l'ordre de $0,7 \mathrm{du}$ paramètre de cavitation du venturi défini par: 


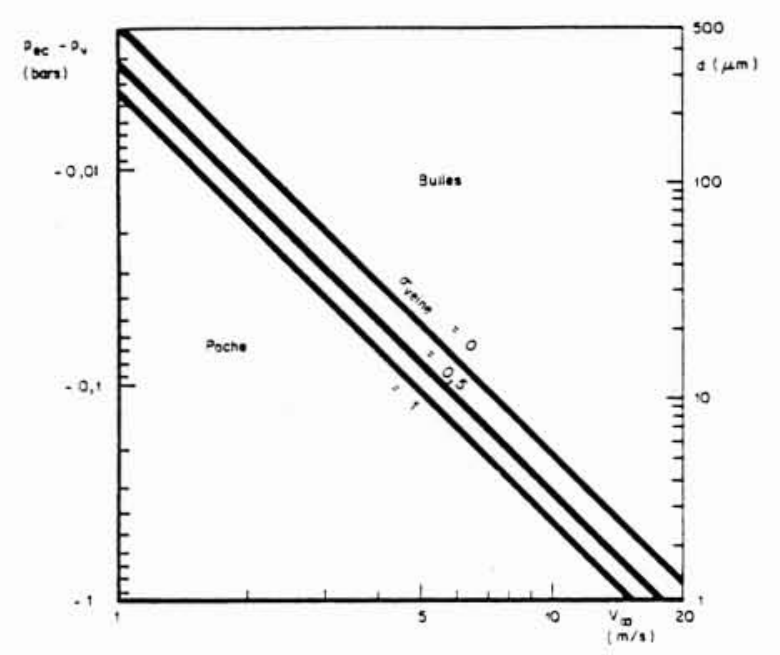

9. Diamètre ou pression d'éclatement minimum mesurable à l'aide du venturi en fonction de la vitesse et du paramètre de cavitation dans la veine d'essais.

$$
\sigma_{\text {venturi }}=\frac{p_{a m}-p_{v}}{1 / 2 \rho V_{e c}^{2}}
$$

où les indices $a m$ et $e c$ sont relatifs respectivement à l'amont du venturi et au point d'éclatement des germes. La condition de cavitation par bulles $\sigma_{\text {venturi }}>\sigma_{1}$ limite la pression d'éclatement minimale conformément à la relation suivante ${ }^{(*)}$ :

$$
p_{e c}-p_{v}>-\frac{1-\sigma_{\ell}}{\sigma \ell}\left(p_{\mathrm{am}}-p_{v}\right)
$$

La pression $p_{a m}$ à l'amont du venturi est fixée par les conditions de fonctionnement du tunnel sur lequel le dispositif est implanté puisqu'elle s'identifie, aux pertes de charge près, à la pression à l'amont du convergent de la veine d'essais. Si le taux de convergence du tunnel est suffisamment grand pour que la pression dynamique amont soit négligeable, la pression amont $p_{a m}$ est donnée en fonction des caractéristiques de fonctionnement en vitesse $V_{\infty}$ et en paramètre de cavitation $\sigma_{\text {veine }}$ dans la veine d'essais par la relation suivante ${ }^{(*)}$ :

$$
p_{a m}-p_{v}=\left(1+\sigma_{\text {veine }}\right) \frac{1}{2} \rho V_{\infty}^{2}
$$

La dynamique de mesure en pression d'éclatement est donc d'autant plus grande que les essais se font à grande vitesse et à paramètre de cavitation élevé.

En pratique, cette limitation vers les basses pressions critiques n'est effective qu'à faible vitesse comme l'indique la figure 9. C'est ainsi que, à la vitesse de $3 \mathrm{~m} / \mathrm{s}$ et pour $\sigma_{\text {veine }}=0,5$ par exemple, les germes dont le diamètre est inférieur à $40 \mu \mathrm{m}$ ne peuvent pas être dénombrés. Cette limite est cependant très vite repoussée lorsque la vitesse augmente : à $10 \mathrm{~m} / \mathrm{s}$, on trouve un diamètre limite de $3,5 \mu \mathrm{m}$.

Remarquons pour terminer que, dans le cas d'essais à faibles vitesses d'écoulement et selon la distance entre la section d'injection et la section d'essais, le temps de séjour des germes peut être suffisamment long pour que les phénomènes de résorption par diffusion ou de tri par gravité deviennent sensibles. Par ailleurs, il est important que le moyen d'essais assure une bonne résorption des bulles à l'aval de la section d'essais afin qu'elles ne recirculent pas et que, en cas d'utilisation de la dilution pour la mesure, celle-ci apporte peu de germes en comparaison avec le prélèvement. Le dispositif d'injection et de mesure de germes constitue donc un ensemble dont les caractéristiques sont dépendantes de celles du moyen d'essais qu'il équipe.

\section{Influence de la nucléation sur la cavita- tion - Résultats récents}

\subsection{Germes, couche limite et cavitation}

La cavitation est fortement dépendante, en plus de l'état de nucléation de l'eau, de l'état de la couche limite comme l'ont montré plusieurs études (voir par exemple Arakeri \& Acosta, 1973 - Van Der Meulen, 1980 Michel \& al., 1982). Or, si l'effet des germes peut être découplé de celui de la viscosité par un traitement approprié de l'eau, l'inverse est évidemment impossible. L'effet des germes sur la cavitation ne peut donc être analysé sans considérer l'état de la couche limite.

En l'absence de germes, la cavitation se manifeste généralement sous la forme d'une poche attachée à la paroi. La poche de cavitation interagit fortement avec la couche limite qui se développe sur la partie mouillée de la paroi en amont du détachement de la cavité. L'interaction entre cavité et couche limite est caractérisée par les deux points suivants (Franc \& Michel, 1985).

D'une part, une cavité se détache derrière un décollement laminaire de couche limite. L'existence d'une zone d'eau "morte " derrière le décollement est en effet déterminante pour l'équilibre mécanique de la cavité.

D'autre part, une cavité attachée ne peut coexister avec une couche limite turbulente. La transition à la turbulence permet à la couche limite de surmonter des gradients adverses de pression bien supérieurs au cas laminaire sans décoller et a ainsi pour effet, en éliminant le décollement, de chasser la poche de cavitation.

L'injection de germes, quant à elle, a tendance à produire un autre type de cavitation, par bulles séparées voyageuses, qui entre en compétition avec la cavitation par poche. Une question importante en pratique est de savoir quel type de cavitation prévaut compte tenu de l'état de nucléation de l'eau et des caractéristiques de l'écoulement.

Des essais menés récemment au CERG et à l'IMG sur des ogives et sur des profils d'ailes tendent à préciser les conditions du passage d'un type de cavitation à l'autre. En particulier, deux expérimentations sont menées en parallèle au CERG et à l'IMG sur la même forme de

(*) Compte tenu du fort rapport de contraction entre section amont et section d'éclatement, la pression dynamique amont est négligée. 


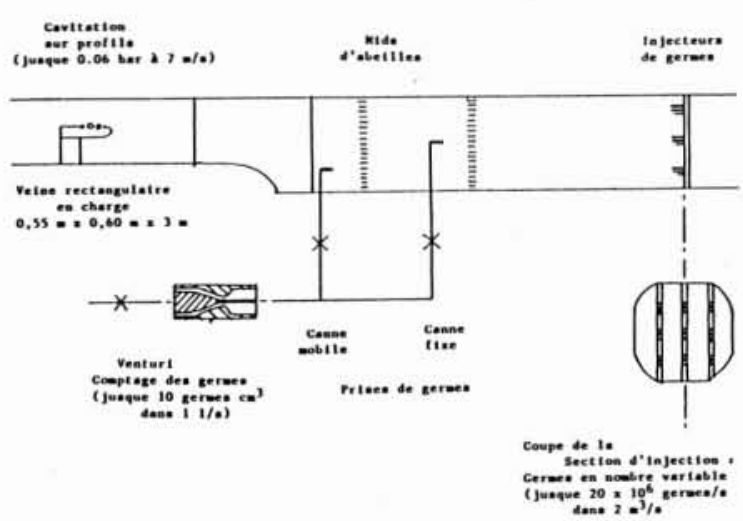

10. Schéma du dispositif de contrôle de germes sur le TH8. Branches $2 \mathrm{~m}^{3} / \mathrm{s}$ du CERG.

profil (type Naca 16-209) mais à deux échelles géométriques différentes. Un profil de corde $10 \mathrm{~cm}$ est essayé à l'IMG et un autre de corde $60 \mathrm{~cm}$ au CERG, pour divers états de nucléation de l'eau, en vue d'analyser l'interaction entre germes, couche limite et cavitation et de préciser en particulier l'effet d'un changement d'échelle géométrique.

\subsection{Résultats obtenus sur la boucle du CERG}

Le tunnel hydrodynamique TH $8\left(8 \mathrm{~m}^{3} / \mathrm{s}\right)$, dans sa branche à $2 \mathrm{~m}^{3} / \mathrm{s}$, qui permet d'étudier la cavitation sur profils, a été récemment (début 1986) équipé des derniers dispositifs d'injection et de mesure de germes mis au point par le CERG (fig. 10).
Des corrélations ont été obtenues entre les spectres de germes mesurés par prélèvement et la cavitation observée sur plusieurs profils : deux ogives et une aile à incidence variable.

Les résultats présentés ici concernent la suppression de poche de cavitation, liée à un décollement laminaire, par l'injection de microbulles d'air dans une eau préalablement dégazée.

Ogive allongée (longueur $\simeq$ diamètre $\simeq 0,1 \mathrm{~m}$ ) ̀̀ incidence nulle

Hors cavitation et pour un Reynolds $R e=0.7 \times 10^{6}$, cette ogive présente un décollement laminaire où le coefficient de pression $C_{p s}=-0.35$.

Lorsque la pression dans le décollement descend en-dessous de la pression de vapeur, il apparaît une poche de cavitation, de plus en plus longue et épaisse. Sans injection de germes et à $\sigma_{v}=0,27$, cette poche est globalement stable (figure 11a).

L'injection de germes tend à supprimer ladite poche. Pour obtenir un coefficient d'intermittence de l'ordre de 0,5 doivent être injectés environ $\mathrm{n} \simeq 0,024$ germes $/ \mathrm{cm}^{3}$ (fig. 11b). La mesure de germes est réalisée par une prise fixe en amont du profil (fig. 10) après qu'on ait contrôlé au préalable l'homogénéité spatiale par une prise mobile. Les germes sont comptés pour une pression au col du Venturi $P_{\mathrm{col}}=-8$ mbar égale à la pression minimale que les bulles voient sur le profil de l'ogive, à partir du $\sigma$ d'apparition des bulles (avec injection maximale de germes) $\sigma_{i b}=0.4$.

Les nombres ainsi obtenus englobent les germes depuis les plus gros, de pression critique quasiment égale à la pression de vapeur, jusqu'à ceux dont la pression critique vaut la pression minimale vue par les bulles sur le profil considéré (inférieure à $p_{v}$ ). t

(a) eau dégaxée sana in jection de germes

(b) avec injection de $0,024 \mathrm{germes} / \mathrm{cm}^{3}$ comptéa
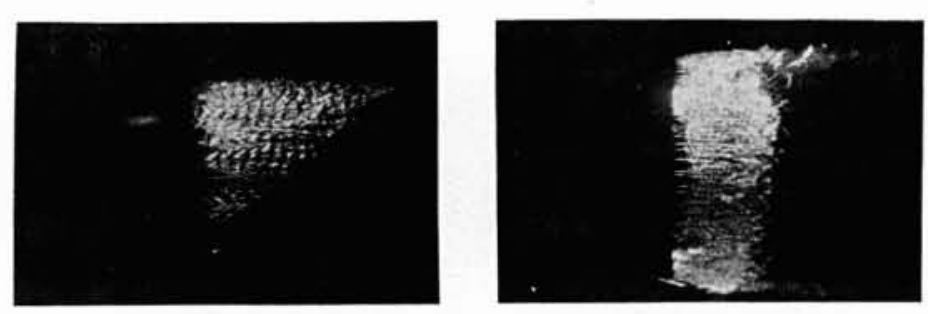

(c) eau dégazée sans injection de germea

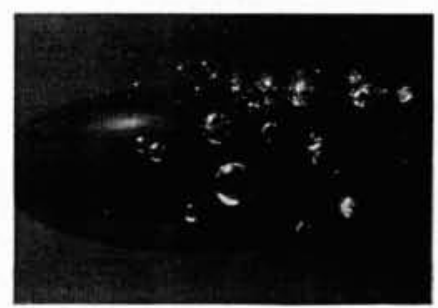

Ogive allongée $0=0,27$

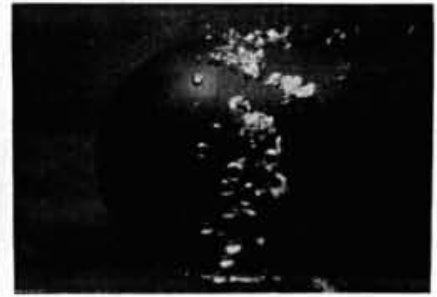

(d) avec infection de 0,7 germes/cm comptés à - 28 mar
Ogive hémisphérique $0=0,85$

11. Figures de cavitation sur ogives. 
Ogive hémisphérique (diamètre $\simeq 0,1 \mathrm{~m}$ ) ̀̀ incidence nulle

De façon analogue à l'ogive allongée, les résultats suivants ont été obtenus :

- Décollement laminaire pour $R e=0,7 \times 10^{6}$ ayant un $C_{p s} \simeq-0,7$

- Sans injection, et à $\sigma=0,6$, poche stable (fig. 11c)

- Intermittence bulles/poche (fig. 11d) pour $\mathrm{n}=0,7$ germes $/ \mathrm{cm}^{3}$ comptés à $P_{\mathrm{col}}=-28 \mathrm{mbar}$ calculé avec $\sigma_{i b}=0,8$.

Le rapport 30 entre l'ogive allongée $(n \simeq 0,024$ ger$\mathrm{mes} / \mathrm{cm})$ et l'ogive hémisphérique $\left(\mathrm{n} \simeq 0,7\right.$ germes $\left./ \mathrm{cm}^{3}\right)$ peut être corrélé à la plus grande taille des bulles sur l'ogive allongée au niveau du détachement de poche, due à une zone de dépression plus étendue. Les photographies révèlent que sur ogives les bulles ont tendance à "balayer" les poches sur leur passage, et que les poches mettent un certain temps à se reformer. Les bulles les plus grosses suppriment plus facilement les poches. Le nombre de bulles joue également, mais, compte tenu du temps de reformation des poches, il n'est pas nécessaire que les bulles occupent tout le profil pour balayer les poches.

Aile à incidence 3,2 degrés (Naca 16-209 corde 0,6 $m$, épaisseur $53 \mathrm{~mm}$ )

Hors cavitation, il se produit sur l'extrados un décollement laminaire environ $3 \mathrm{~cm}$ après le bord d'attaque, avec un $C_{p s} \simeq-0.88$.

Sans injection, et à $\sigma=0,78$ (fig. 12a) la cavitation se manifeste sous forme d'une rangée de poches ("doigts »), moins stables que les poches obtenues sur ogives.

L'intermittence bulles/poches est obtenue pour $n \simeq 1$ germes $/ \mathrm{cm}^{3}$ (fig. 12b), comptés à $P_{\mathrm{col}}=-12 \mathrm{mbar}$, calculé avec $\sigma_{i b} \simeq 0.96$.

\subsection{Résultats obtenus sur la boucle de l'IMG}

Les résultats présentés dans ce paragraphe sont relatifs aux essais menés à l'IMG sur l'aile de type Naca 16-209 de corde $10 \mathrm{~cm}$.

En l'absence de germes, la cavitation à l'extrados de l'aile se présente sous la forme d'une cavité dont le détachement peut prendre plusieurs configurations selon la valeur de l'angle d'incidence $\alpha$ et du paramètre de cavitation $\sigma_{v}$ comme le montre le diagramme de la figure 13.

\section{Domaine 1}

Aux faibles incidences $\alpha \widetilde{<} 3$ deg. et pour les faibles nombres de Reynolds et de taux de turbulence obtenus dans le tunnel de l'IMG, la couche limite en régime non cavitant est laminaire et décolle à l'arrière du profil. Aux faibles valeurs du paramètre de cavitation $(\sigma v \simeq 0,2)$, une cavité se détache de manière bidimensionnelle derrière le décollement laminaire dont la position se trouve d'ailleurs légèrement affectée par le développement de la cavité.

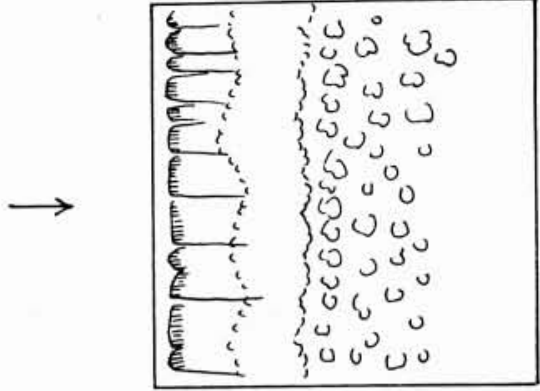

(a) Eau dégazée, sans injection de germes

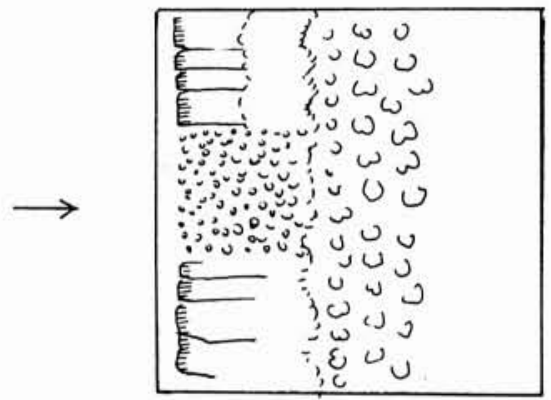

(b) avec injection de $\simeq 1$ germe $/ \mathrm{cm}^{3}$ comptés à $P_{\text {col }} \simeq-12$ mbar

12. Figures de cavitation sur aile (CERG).

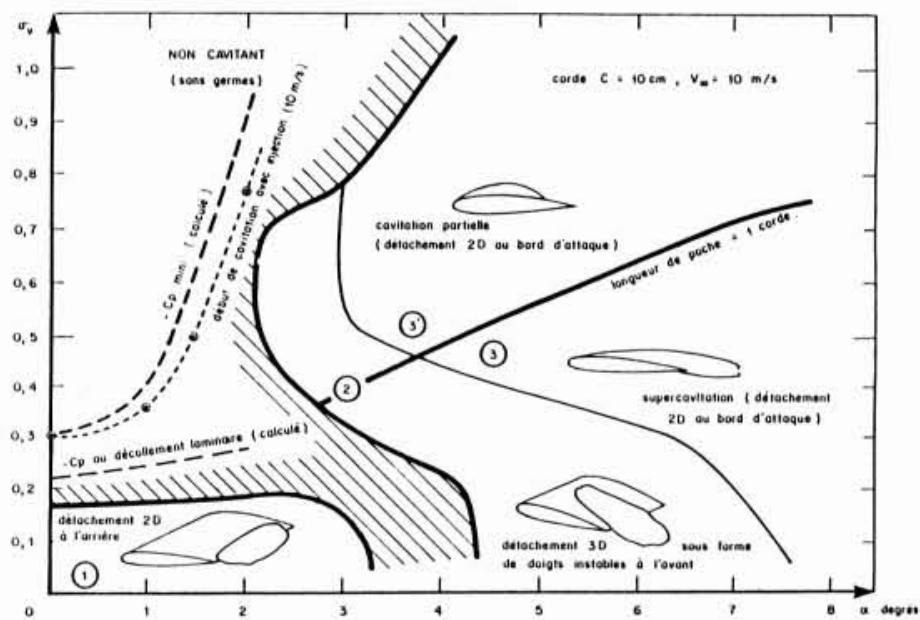

13. Types de cavitation observés à l'IMG sans injection sur l'extrados du profil Naca 16.209.

Le décollement laminaire a pu être mis en évidence grâce à une injection ponctuelle de colorant au bord d'attaque qui permet de visualiser la couche limite et de localiser ses principaux points remarquables tels que les points de transition et de décollement. 


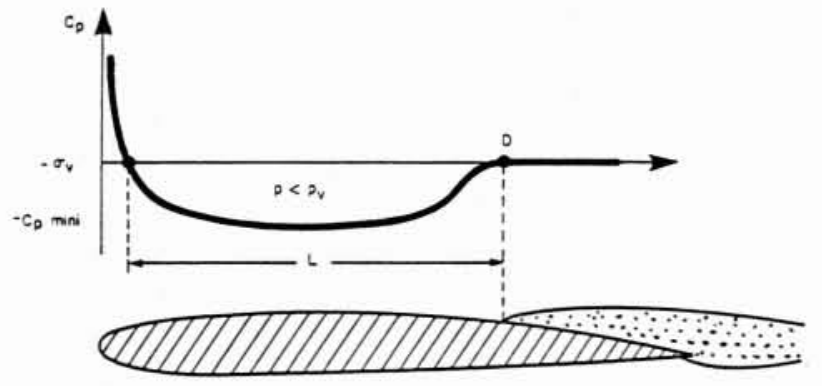

14. Allure de la répartition de pression en cavitation à poche.

La couche limite ne peut décoller que si elle remonte un gradient adverse de pression. La pression dans la cavité étant très voisine de la pression de vapeur saturante, il existe nécessairement une zone de pression inférieure à la pression de vapeur en amont de la cavité. Cela prouve en particulier que la cavitation par poche apparait pour une valeur du paramètre de cavitation $\sigma_{v}$ inférieure $\grave{a}-C_{p \operatorname{mini}}$ et de l'ordre de l'opposé du coefficient de pression au décollement. Dans le cas des profils minces tels que celui étudié ici, à faible incidence, la zone de dépression $p<p_{v}$ s'étend sur la plus grande partie de zone mouillée en amont du détachement (fig. 14).

Si l'eau contient des germes, ceux dont la pression critique est supérieure à la pression minimum sont activés lorsqu'ils traversent cette zone de dépression et donnent naissance à des bulles de cavitation. L'expérience montre que les deux types de cavitation, par poche attachée et par bulles séparées, coexistent tant que la concentration en germes actifs ne dépasse pas une valeur relativement faible de l'ordre de 0,15 germes $/ \mathrm{cm}^{3}$ à l'incidence de 0 deg. et pour les conditions d'essais suivantes réalisées sur le tunnel de l'IMG: $\sigma_{v}=0.056$ et $V_{\infty}=10 \mathrm{~m} / \mathrm{s}$. Au-delà de ce seuil, la poche est destabilisée et disparaît au profit d'une cavitation par bulles séparées voyageuses.

En ce qui concerne les essais menés au CERG sur l'aile de même forme mais de corde $60 \mathrm{~cm}$, le nombre de Reynolds est nettement supérieur (de l'ordre de $5.10^{6}$ ) ainsi que le taux de turbulence général de l'écoulement. Il s'ensuit que, dans le domaine 1 , la couche limite transitionne à la turbulence et que aucun décollement ni aucune poche de cavitation attachée ne peut exister. Le seul type de cavitation qui peut alors apparaitre est une cavitation par bulles, quel que soit l'état de nucléation.

\section{Domaines 3 et $3^{\prime}$}

Les phénomènes qui apparaissent dans les domaines 3 et $3^{\prime}$ sont qualitativement identiques à ceux qui viennent d'être présentés concernant le domaine 1 . Sans injection de germes, la cavitation se manifeste sous la forme d'une poche qui se détache de manière bidimensionnelle du bord d'attaque. Selon les valeurs de l'incidence et du paramètre de cavitation, la cavité est plus ou moins développée, sa longueur pouvant varier d'une fraction de la corde (cavitation partielle) à la corde ou plus (super- cavitation). La couche limite est laminaire et décolle devant le point de détachement de la cavité.

La différence essentielle par rapport au cas d'une cavité de bord de fuite est que la zone de dépression qui conduit la couche limite à décoller et une cavité à se détacher est due à un pic de dépression profond et localisé au bord d'attaque. De ce fait, les principaux points caractéristiques (point d'arrêt, de pression minimum, de décollement et de détachement) se trouvent rassemblés au bord d'attaque alors qu'ils étaient répartis le long de l'aile dans le cas des faibles incidences.

Comme précédemment, l'injection de germes peut conduire à la disparition de la poche. Cependant, la concentration en germes actifs nécessaire au passage d'une cavitation par poche à une cavitation par bulles est plus grande comme nous l'expliquerons au paragraphe 5. Les essais menés au CERG sur la grande aile (voir $\S 4.2$ ) ont montré que pour une incidence de 3,2 deg., une vitesse de $7 \mathrm{~m} / \mathrm{s}$ et un paramètre de cavitation 0,86 , la valeur seuil pour la concentration en germes actifs est de l'ordre de 1 germe $/ \mathrm{cm}^{3}$. Si on transpose cette valeur au cas des essais menés à l'IMG à l'échelle $1 / 6$ conformément à la règle de similitude en $\lambda^{3}$, on obtient une valeur seuil très grande de l'ordre de 216 germes $/ \mathrm{cm}^{3}$ qui ne peut pas être atteinte en pratique.

\section{Domaine 2}

Il s'agit d'un domaine de transition entre les régimes de cavité de bord d'attaque 3 et 3 ' et le régime non cavitant dans lequel la couche limite transitionne à la turbulence sans décoller. En l'absence de germes, le profil peut présenter une poche généralement instable, très fluctuante et très marquée par des effets tridimensionnels. $\mathrm{La}$ moindre perturbation, quelques germes qui explosent par exemple, la fait disparaître.

\section{5. Éléments d'analyse physique de l'effet des germes sur une poche de cavitation}

Compte tenu des résultats expérimentaux qui viennent d'être présentés et de quelques autres études (en particulier Gates \& Acosta, 1978), plusieurs mécanismes peuvent être avancés pour tenter d'expliquer le passage d'une cavitation par poche attachée à une cavitation par bulles séparées sous l'effet d'une injection de germes.

Il s'agit de mécanismes indirects dans la mesure où ils affectent, au premier chef, la couche limite, qui par ailleurs est supposée gouverner directement le type de cavitation.

Sous cette hypothèse, une poche de cavitation ne peut disparaitre que si le décollement laminaire qui la précède est inhibé. Deux mécanismes qui conduisent à la disparition du décollement laminaire peuvent être envisagés.

\section{Mécanisme 1}

Les germes, en explosant, peuvent déstabiliser la couche limite comme l'ont montré Gates \& Acosta (1978). Ces auteurs ont mis en évidence sur des corps hémisphériques que, lorsque le nombre de germes injectés augmente, le décollement laminaire devient progressivement 
instable et peut même être complètement éliminé, conciuisant une cavitation par bulles à remplacer une cavitation attachée.

Les mécanismes détaillés selon lesquels la couche limite est déstabilisée par les germes n'ont pas encore été mis en évidence. Cependant, on peut imaginer que, du fait des vitesses radiales induite par l'explosion d'une bulle, le décollement laminaire et la cavité attachée qu'il abrite disparaissent temporairement soit par un effet de souffle, soit par un effet d'excitation de la transition à la turbulence. On a observé en effet (voir §4.2) que le passage d'une bulle peut créer localement un trou dans un cavité attachée. $\mathrm{Si}$ le temps caractéristique de rétablissement du décollement laminaire et de la poche est supérieur au temps caractéristique de la fréquence de passage des bulles, la cavité attachée n'a pas le temps de se rétablir et est remplacée par une cavitation par bulles.

Une des caractéristiques de ce mécanisme est que la répartition de pression n'est modifiée que d'une manière locale temporaire.

\section{Mécanisme 2}

Si la couche limite n'est pas déstabilisée par l'explosion de germes, même en grand nombre, un deuxième mécanisme qui conduit à une modification permanente de la répartition de pression prend le relai du premier.

La pression est modifiée par la présence de bulles de cavitation macroscopique dans la mesure où la pression dans une bulle est égale à la pression de vapeur. Selon les conditions d'injection et d'écoulement, il peut exister un point $S$ sur le profil, dit point de saturation, en aval duquel les bulles viennent en contact. En aval de ce point, la pression se trouve nivelée à $p_{v}$ (voir fig. 15). Plus la concentration en germes actifs est élevée, plus le point de saturation $S$ remonte vers l'amont.

Considérons le cas d'une poche de cavitation se détachant du point $D$ comme indiqué sur la figure 14. La zone de dépression en amont du détachement fait grossir les germes. Il existe une valeur critique de la concentration en germes actifs (qui est estimée ci-dessous) pour laquelle la saturation est atteinte au détachement de la cavité. Pour une concentration inférieure, la répartition de pression sur le profil est peu changée par les bulles de cavitation, du moins d'un point de vue stationnaire, alors que pour une concentration supérieure le palier $p=p_{v}$ démarre d'un point de saturation $S$ situé en amont du point de détachement $D$. Dans ce dernier cas, la zone de gradient adverse est moins importante et devient insuffisante pour que la couche limite décolle (Franc \& Michel, 1985). Cette concentration critique est donc la concentration limite à partir de laquelle la poche de cavitation disparaît au profit de bulles séparées. Elle peut être estimée comme suit.

La couche de liquide (épaisseur $\delta$ ) dans laquelle la pression est inférieure à $p_{v}$ et qui contient par conséquent les germes activés par la zone de dépression peut être estimée à partir de l'équation d'Euler normale au profil :

$$
\left|\frac{d p}{d n}\right|=\rho \frac{V^{2}}{r}
$$

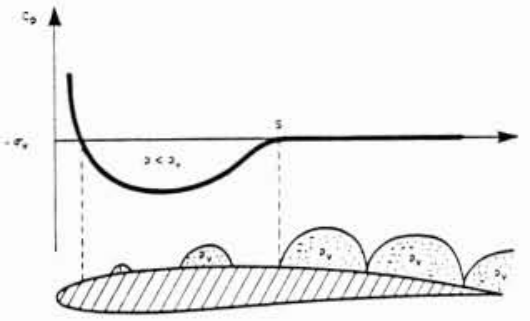

15. Saturation en cavitation par bulles.

dans laquelle $r$ désigne un rayon de courbure. En choisissant $\frac{p_{v}-p_{\operatorname{mini}}}{\delta}$ comme ordre de grandeur du gradient normal de pression $|d p / d n|$ et en assimilant $r$ au rayon de courbure moyen du profil dans la zone de dépression, on obtient l'estimation suivante de $\delta$ :

$$
\frac{\delta}{r} \simeq \frac{-C_{p \operatorname{mini}}-\sigma_{v}}{2\left(1-C_{p \operatorname{mini}}\right)}
$$

Si la concentration volumique en germes actifs est $n$, la densité $\eta$ de bulles de cavitation par unité de surface du profil est, en première approximation $\eta \simeq n \delta$.

En fin de croissance, les bulles atteignent un rayon $R_{\max }$ dont l'ordre de grandeur est donné par la formule (5)

$$
\frac{R_{\max }}{L} \simeq \sqrt{\frac{-C_{p \operatorname{mini}}-\sigma_{v}}{3\left(1-C_{p \operatorname{mini}}\right)}}
$$

dans laquelle $L$ désigne la longueur de la zone de dépression. Cette relation ne tient pas compte des interactions entre bulles.

S'il y a saturation en fin de croissance, la densité surfacique $\eta$ de bulles est de l'ordre de $\frac{1}{\pi R_{\max }^{2}}$, d'où l'ordre de grandeur de la concentration volumique critique en germes actifs :

$$
n \simeq \frac{6}{\pi}\left(\frac{1-C_{p \operatorname{mini}}}{-C_{p \min }-\sigma_{v}}\right)^{2} \frac{1}{r L^{2}}
$$

Outre le coefficient de pression minimale $C_{p \operatorname{mini}}$ et le paramètre de cavitation $\sigma_{v}$, cette formule fait intervenir le rayon de courbure moyen $r$ du profil dans la zone de dépression et la longueur $L$ de cette zone. On retrouve sur cette formule la règle de similitude en $\lambda^{3}$ énoncée au paragraphe 2.2. L'attention du lecteur est attirée sur le fait que les paramètres $C_{p \operatorname{mini}}$ et $L$ sont relatifs à l'écoulement en présence de la poche de cavitation.

Compte tenu des approximations grossières qui ont été faites, on ne peut attendre des formules précédentes que des ordres de grandeur ou des tendances générales. Dans le cas du profil Naca 16.209 de corde $c=10 \mathrm{~cm}$, 
pour $\sigma_{v}=0,056$ et à l'incidence $\alpha=0$ deg., on a les ordres de grandeurs suivants :

- longueur de la zone de dépression $L \simeq 75 \mathrm{~mm}$

- coefficient de pression minimale $C_{p \operatorname{mini}} \simeq-0,2$

- rayon de courbure moyen du profil dans la zone de dépression $r \simeq 450 \mathrm{~mm}$

- épaisseur de la couche de liquide contenant les germes activés $\delta \simeq 27 \mathrm{~mm}$

- rayon des bulles en fin de croissance $R_{\max } \simeq 15 \mathrm{~mm}$

- concentration critique en germes actifs $n \simeq 0,05$ germe $/ \mathrm{cm}^{3}$

Les ordres de grandeur précédents sont cohérents par rapport à l'expérimentation. En particulier, la concentration critique en germes actifs avait été estimée à 0,15 germe $/ \mathrm{cm}^{3}$ à partir des essais.

Dans le cas d'une cavité de bord d'attaque, à plus forte incidence, les formules précédentes montrent que la concentration en germes nécessaire pour chasser la poche est supérieure par plusieurs ordres de grandeur. En effet, d'une part la zone de dépression qui est due à un pic très localisé a une longueur $L$ faible, ce qui laisse peu de temps aux bulles pour grossir. D'autre part, le rayon de courbure $r$ du profil au niveau du pic de dépression situé au voisinage du bord d'attaque est petit; par conséquent, l'influence du pic de dépression décroît rapidement normalement au profil et seuls les germes qui passent au voisinage immédiat de la paroi peuvent être activés.

La formule (9) donne l'ordre de grandeur de la concentration critique en germes au-delà de laquelle la cavitation se manifeste nécessairement sous la forme de bulles séparées et non d'une poche attachée, du fait d'une modification de la répartition de pression. Il se peut cependant que, selon les conditions d'écoulement, d'autres mécanismes, tels que le déclenchement de la turbulence dans la couche limite ou un effet de souffle dû à l'explosion des germes, provoquent une transition d'une cavitation par poche attachée à une cavitation par bulles séparées pour une concentration en germes moindre.

\section{Conclusion}

Parmi les nombreux facteurs qui influent sur la cavitation, la qualité de l'eau joue un rôle fondamental en raison des germes qu'elle peut contenir. Il est donc indispensable en pratique de contrôler la teneur en gaz libre et en gaz dissous de l'eau du moyen d'essais.

Le dispositif mis au point au CERG permet d'injecter des microbulles d'air en quantité variable à l'amont de la veine d'essais et de mesurer au moyen de venturi spéciaux le nombre et la dimension des germes ainsi créés.

De nombreux essais réalisés récemment dans des configurations variées (ogives et ailes bidimensionnelles) sur les installations du CERG et de l'IMG équipées d'un tel dispositif ont permis de mettre en évidence l'influence de l'état de nucléation de l'eau et des caractéristiques de l'écoulement sur le type de cavitation naissante ou développée.

En particulier, nous avons observé que la présence de germes de cavitation dans l'eau conduit une cavitation par bulles séparées à remplacer une cavitation par poche attachée si les deux conditions suivantes sont satisfaites. D'une part, les germes doivent être effectivement activés par la zone de dépression qui précède la poche ce qui nécessite qu'ils soient suffisamment gros pour que leur pression critique soit supérieure à la pression minimale de l'écoulement. D'autre part, la concentration en germes actifs doit dépasser une valeur critique qui, à l'heure actuelle, ne peut être connue précisément que par voie expérimentale.

Ces recherches ont été soutenues en grande partie par la Direction des Recherches, Etudes et Techniques (Contrats DRET 84.067 et 84.465 ). 


\section{Références}

[1] ARAKERI V.H. \& ACOSTA A.J., 1973. - Viscous effects in the inception of cavitation on axisymmetric bodies. Journal of Fluids Engineering, 95, 519-527.

[2] ARndT R.E., 1981. - Cavitation in fluid machinery and hydraulic structures. Annual Review of Fluid Mechanics, $13,273-328$.

[3] Avellan F., Gindroz B., Henry P., Bachmann P., Vullioud G., \& WEGNER M., 1986. - Influence de la chute d'essai et de la nucléation sur les performances en cavitation des modèles de turbines Francis. Symposium AIRH, Montréal.

[4] BiLlet M.L., 1985. - Cavitation nuclei measurements. A review. Cavitation and multiphase Flow Forum.

[5] Franc J.P. \& Michel J.M., 1985. - Attached cavitation and the boundary layer: experimental investigation and numerical treatment. Journal of Fluid Mechanics, 154, 63-90.

[6] Gates E.M. \& Acosta A.J., 1978. - Some effects of several freestream factors on cavitation inception of axisymmetric bodies. Proceedings of the $12^{\text {th }}$ Symposium on Naval Hydrodynamics, Washington D.C., 86-112.

[7] Henry P., Lecoffre Y. \& Laborde P.Y., 1980. - Scale effects of cavitation phenomena. Symposium AIRH, Tokyo.

[8] Holl J.W. \& Wislicenus G.F., September 1961. - Scale effects on cavitation. Journal of Basic Engineering, 385-398.
[9] LECOFfre Y., 1986. - Méthodes pratiques de contrôle de la teneur en air libre et dissous dans les moyens d'essais. Symposium AIRH, Montréal.

[10] LE GoFF J.P. \& LeCofFre Y., 1982. - Nuclei and cavitation. Proceedings of the $14^{\text {th }}$ Symposium on Naval Hydrodynamics.

[11] Lindgren H. \& Johnsson C.A., 1966. - Cavitation inception on headforms, ITTC comparative experiments. Proceeding of the $11^{\text {th }}$ International Towing Tank Conference, Tokyo, 219-232.

[12] Michel J.M., Biset S. \& Franc J.P., 1982. - Cavitation développée sur des parois à courbure continue : physique du détachement. La Houille Blanche, 7/8, 559-570.

[13] Oldenziel O.M. \& TeiJema, 1976. - Cavitation on valves in correlation to liquid properties. AIRH Symposium, Leningrad, 1976.

[14] Plesset M.S. \& Prosperetti A., 1977. - Bubble dynamics and cavitation. Annual Review of Fluid Mechanics, 9, 145-185.

[15] SUHRBIER K.R. \& LECOFFRE Y., 1986. - Investigation of the influences of test techniques, water speed and nuclei seeding on the characteristics of a high speed model propeller in a cavitation tunnel and correlation with full scale measurements. International Symposium on Cavita. tion, April 1986, Sendai, Japan.

[16] VAN Der Meulen J.H.J., 1980. - Boundary layer and cavitation studies of Naca 16-012 and Naca 4412 hydro. foils. Proceedings of the $13^{\text {th }}$ Symposium on Naval Hydro. dynamics, Tokyo, 195-219.

\section{Discussion}

Président : M. R. SÉMÉRIA

\begin{abstract}
Le Président. - $\mathrm{Y}$ a-t-il des questions sur cette présentation?

M. REALI. - Je voudrais signaler la possibilité de noyaux liquides de gaz carboniques $\mathrm{CO}_{2}$ dans l'eau à température inférieure à la température critique du $\mathrm{CO}_{2}\left(T_{\mathrm{c}}=31,1^{\circ} \mathrm{C}\right)$. Certains résultats expérimentaux seront présentés à l'occasion de la $8^{\mathrm{e}}$ table ronde sur les transitoires hydrauliques (Madeira, 14 au 17 septembre 1987).
\end{abstract}

M. BONNIN. - Les dispositifs d'acquisition d'histrogrammes de germes d'une part, et d'injection de germes d'autre part, sont-ils ou seront-ils un jour adaptés à des liquides autres que l'eau (métaux liquides, eau chaude, $\mathrm{CO}_{2}$ liquide, hydrocarbures, cryogéniques)?

Le Président. - Cette question a déjà été abordée ce matin.

M. LECOFFRE. - Le procédé présenté est adapté à des mesures de nucléation hétérogène dans les liquides lorsque la température est très inférieure à la température critique. L'eau froide est évidemment le cas le plus favorable, mais de telles mesures sont envisageables par exemple dans les métaux liquides. Des essais ont d'ores et déjà été réalisés en sodium liquide au CEA et à l'EDF.

Actuellement, il est difficle de prévoir les limites du champ d'application de la méthode pour un liquide donné; à l'occasion d'essais effectués au CENG, on a trouvé pour l'eau une limite située au voisinage de $120^{\circ} \mathrm{C}$. Au-delà, le régime d'apparition des bulles était différent et les phénomènes sont probablement mieux analysés par des méthodes différentes telles que celle décrite par M. LEBLOND.

M. LEBLOND. - Les régimes de croissance des bulles dans un liquide surchauffé sont très différents selon que la température est très inférieure ou peu inférieure à la température critique. A basse température, le grossissement est très rapide, ainsi l'ébullition est détectée dès que la densité des bulles devient de l'ordre d'une bulle par $\mathrm{cm}^{3}$; chaque bulle peut donc être analysée individuellement et la méthode proposée par M. LECOFFRE est parfaitement adaptée. Par contre, dans un liquide chaud, le grossissement des bulles est contrôlé par la diffusion de la chaleur; le grossissement est lent et l'ébullition n'est détectée que lorsque l'on atteint une densité importante de bulles de faible diamètre, de l'ordre de 1000 bulles par $\mathrm{cm}^{3}$; chaque bulle ne peut plus être détectée individuellement; une technique d'analyse par diffusion de la lumière semble ici plus adaptée. Qu'en pense M. LECOFFRE?

M. LECOFFRE. - Je suis d'accord.

Le Président. - On a montré les limites, mais aussi l'utilité des dispositifs venturis pour quantifier les germes dans les systèmes. En cavitation, cela constitue un progrès important.

Nous allons passer à la troisième communication, qui sera présentée par M. BERTHOUD, du Service des transferts thermiques du Centre d'études nucléaires de Grenoble. 